\title{
Board 58: Work in Progress: Simulated Electrical Schematic of PLC
}

Mr. Ahmed Alothman,

Mr. Majed Abdullah Alyami, Western Kentucky University

Mr. Timothy Alexander Goodwin, Western Kentycky University

Born and raised in Kentucky by a family of teachers, I developed a good skill set of leadership and communication. My father who is a middle school math teacher would have me board race his students when I was in the first and second grade. I have also been involved in music for a very long time; taking guitar lessons for 10 years and also playing saxophone throughout high school and college marching band. My dream is to eventually join along my family and become a professor after a long career in industry.

Miss Courtney Smith, Western Kentucky University 


\title{
Work in Progress: Simulated Electrical Schematic of PLC
}

\author{
Ahmed Alothman, Majed Alyami, Timothy Goodwin, and Courtney Smith \\ Faculty Advisor, Dr. Greg Arbuckle \\ School of Engineering and Applied Sciences \\ Western Kentucky University \\ Bowling Green, Kentucky 42101-3576
}

\begin{abstract}
PLCs control how the world works around us and will continue to for the foreseeable future. Industry has used them for manufacturing lines and sorting devices heavily in the last couple decades with very good results. With such a large amount of implementation throughout the world, initial setup and troubleshooting PLCs is still very difficult. The task of our project would be to create a simulated electrical schematic of the PLC system in operation using LabVIEW. This schematic would simulate ladder logic from Siemens TIA Portal and a physical simulation with Factory I/O. This would create a full simulation with all three phases of the PLC. Overall this project would provide a basis for others to recreate what we will do for their larger systems. This would cut setup and troubleshooting times down tremendously. Any solution could be tested and implemented knowing it would work from the comfort of a desktop.
\end{abstract}

Keywords-PLC; OPC Servers; LabVIEW; Factory I/O; Siemens TIA Portal

\section{INTRODUCTION}

PLCs, or Programmable Logic Controllers, have been used frequently in industry for many years and will continue as long as automation is important to industry. With the future of PLC's very certain there are still efforts to try and simplify the initial installation and troubleshooting of these devices. While there are schematics available, the complex setups of most PLC's make it almost impossible to pinpoint the problem without a major dive into the large set on pins on the device. In the last decade however, there has been a major push to develop communication between PLC's and computers because added connectivity allows more and varied control methods and simulation. This helps ease the ever-changing needs of a factory floor. When a change needs to be added, the team can change all the registers in a simulation, test, and release the changes to the physical PLC all from the comfort of a computer screen. The major updates that are happening with our project that haven't been done before in other research is how detailed the LabVIEW schematic will be. A full application of connectivity will be the primary delivery of this project. Most other projects that have dealt with connection of LabVIEW to PLC variables have stopped with just proof of concept, however the final goal of ours is to design the entire system in LabVIEW. As a demo of the proposed system, we are hoping to initially just turn on a light, but move to more complex systems. Our project is to create a fully automated simulation of three programs: Siemens TIA Portal "Fig. 1", Factory IO "Fig. 2", and LabVIEW "Fig. 3". Siemens TIA Portal is where we write our ladder logic for PLC. This uses inputs, outputs, counters, timers, etc. to accomplish our task. In our case, our Factory I/O simulation will use the ladder logic created in TIA Portal to run a simple box sorter. Factory IO has several layouts of different manufacturing systems from box sorting to paint mixing. We can create our own layout, if we wish to do so, using the conveyors, lights, sensors necessary. In our case, we use a box sorting by height layout. Communication between ladder logic and a simulation such as Factory IO is easy to do, so the new part we are bringing into this communication is with an added communication with National Instruments LabVIEW. LabVIEW will be used to create a fully simulated electrical schematic of the system. When all three programs are running simultaneously, you will be able to see the inputs and outputs light up on the ladder logic in Siemens TIA Portal, the simulation running as it should on Factory IO, and the outputs being seen on LabVIEW. Using LabVIEW, we will also be able to control the inputs as we would on the Factory IO panels. This project will give people the ability to easily and quickly access, update, and control their system from any network connected computer.

\section{PROCESS}

To begin our project properly, the entire team had to be comfortable with all three programs main programs used. We took the first two weeks to play around and research Siemens TIA Portal, Factory IO, and LabVIEW. TIA Portal and Factory IO took some time but overall were not as difficult to deal with. LabVIEW, however, required a lot more attention from us, and after getting comfortable with TIA Portal and Factory IO we spent more time researching and practicing LabVIEW function. We began creating a schematic in LabVIEW that had the goal of turning on a light when the start button was pushed, turned off when the stop button was pushed, and prevented the light from turning on when the E-stop button was pushed. This gave us firsthand experience using some things LabVIEW had to

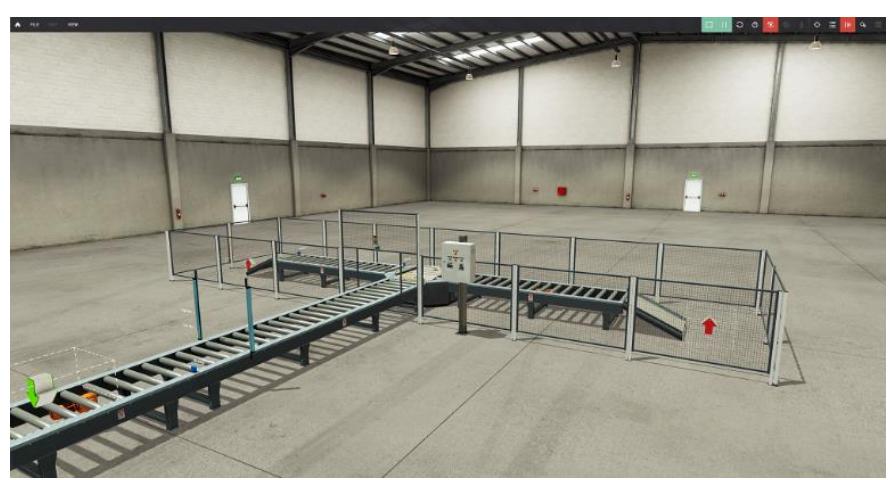

Fig. 1 Factory I/O PLC Simulation Software 


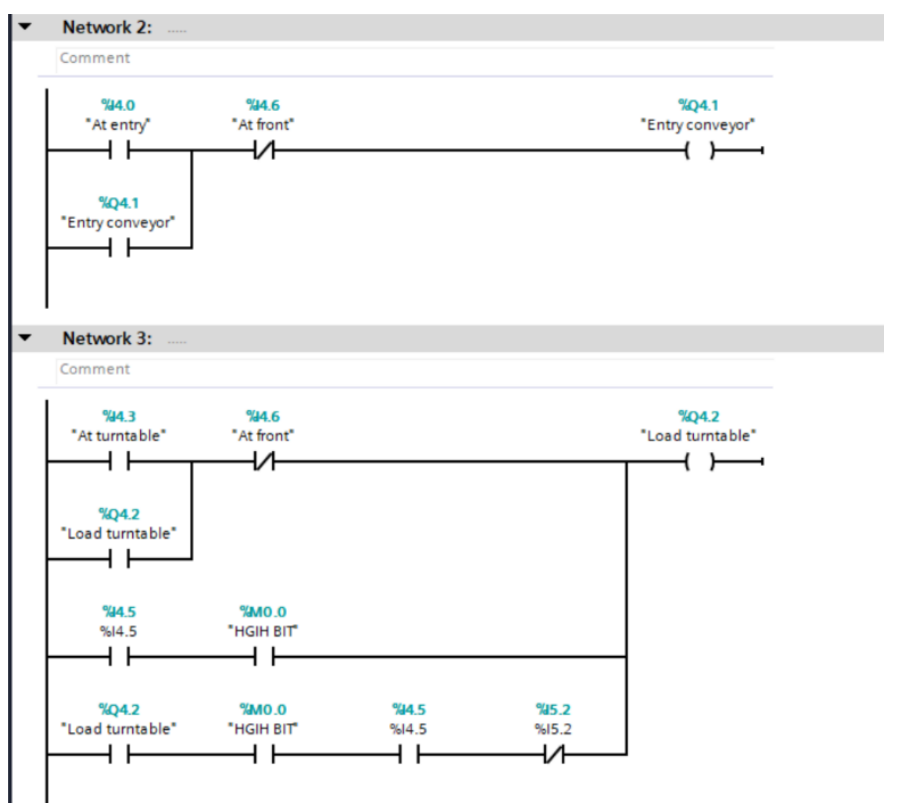

Fig. 2 Siemens TIA Portal Ladder Logic

offer. As we became more experienced with LabVIEW, we began thinking of ways to connect LabVIEW variables to the PLC inputs and outputs. We found many forums of people discussing how to connect LabVIEW with their PLC. Reading through those, we discovered that there is a software known as Modbus that was free and easily downloaded as a LabVIEW add-on. After downloading Modbus and going through the steps to connect to our PLC, we couldn't figure out a way using the Modbus to connect TIA Portal to LabVIEW. We did however learn that Modbus could support the connection of LabVIEW to Factory I/O using a special driver. This new driver took the PLC out of the system making it not useful for our application, but still showed the first step in connecting out software to the PLC. Our next step in trying to connect variables from LabVIEW to the PLC was to try and use Object Linking and Embedding for Process Control (OPC) servers to connect LabVIEW variables straight to the PLC input and output variables using object linking. After downloading the needed software add-ons from LabVIEW the server needed to be set up. This took some time as getting all of the settings correct were difficult as the software needed to track all of the variables through different variables linked through each computers I.P. address. With the OPC server set up it would consistently update LabVIEW variables using a while loop that iterates consistently at a specified rate of our choosing. For us $100 \mathrm{~ms}$ made the most sense as this was the standard loop time in LabVIEW as well. This server turned out to be exactly what we were looking for, however, the computer we were using couldn't handle all the programs and communications running at the same time without lagging significantly so we had to get a more powerful computer. Transferring all of the software licenses put another hamper on time for the project. Once all of the software was available we connected TIA Portal, Factory IO, and LabVIEW together. This was the breaking point for our project. Once all of the variables were linked using OPC servers we started right away on the LabVIEW control panel that would

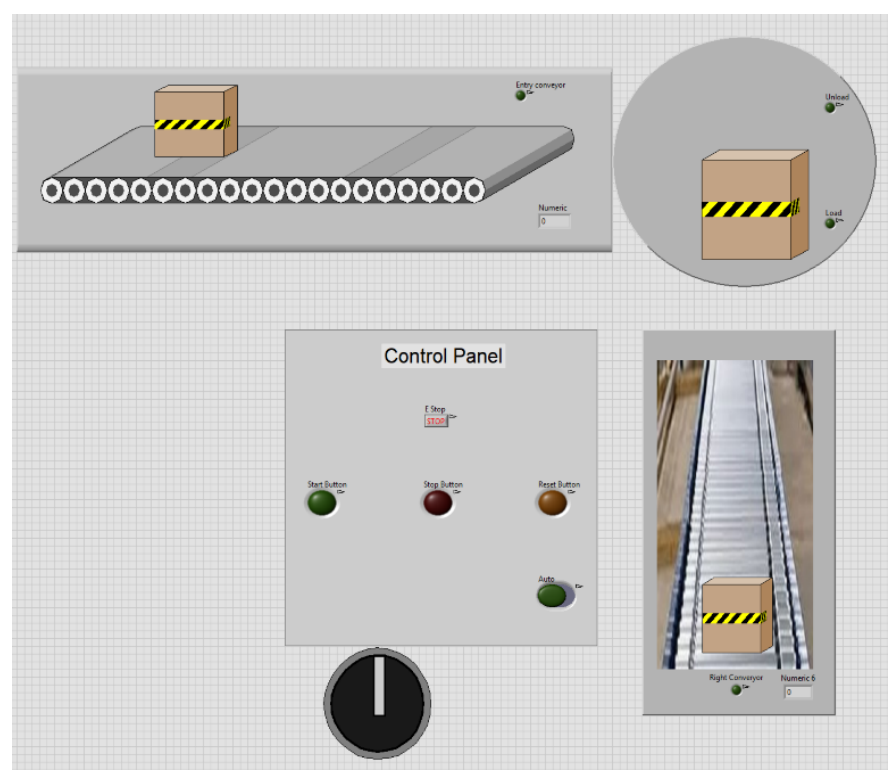

Fig. 3 LabVIEW Control Panel

both simulate the box sorter visually and with an electrical schematic in the background. Once the visual simulation was complete in LabVIEW we had to work on the schematic that would be working in the background, making the visuals correct. We did this by sending the LabVIEW schematic to Multisim and back to simulate the motors and relays. In parallel with the LabVIEW being close to completion, there was an effort to make a physical simulation of the box sorter using the PLC Trainer outputs. With a ladder logic program running we can not only simulate the program with Factory I/O but with a physical motorized setup. $24 \mathrm{~V}$ dc motors were bought because they would work with the PLC outputs without conversion and 3D printed stands and turntable with bands made up the conveyers. Ice Cube relays were also used as motor starters and

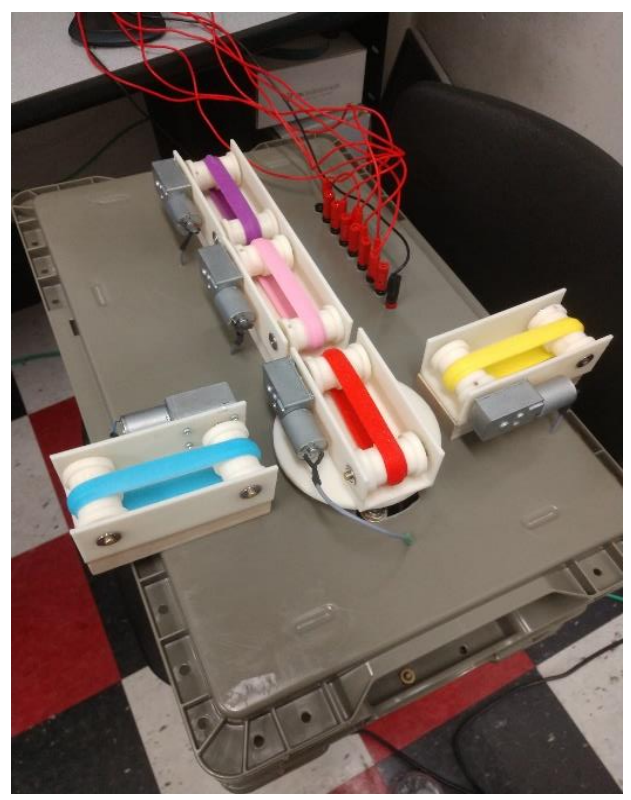

Fig. 4 Physical Simulation of Box Sorter 
the PLC Trainer outputs as the controller which allows us both synthetic and physical simulation of a PLC system. A picture of the physical simulation can be found in "Fig. 4". With the physical simulation we have simulated the entire PLC system. Implementation to a full system would be trivial.

\section{CONCLUSIONS}

The first result that we found was the connection of LabVIEW and Siemens PLC was possible through several different methods with OPC servers being the most reliable. With the connection a possibility, we have done preliminary prototyping and proof of concept with a Factory I/O control panel recreated in LabVIEW for a simple box sorter by height. This control is the key to developing a full electrical schematic in LabVIEW as all of the inputs and output could be modeled using the new OPC variables. The last result that was finished in early march was an added physical simulation. The PLC trainer we have been using has $24 \mathrm{~V}$ outputs that were perfect with some small motors we bought. We are currently working on completing the full electrical schematic in LabVIEW, which would allow a PLC system to have a fully simulated electrical schematic. This would allow PLC systems to be troubleshot and have the implementation of solutions all to be done with just a few keystrokes and mouse clicks.

\section{ABBREVIATIONS AND ACRONYMS}

PLC: Programmable Logic Controller

OPC: OLE for Process Control

OLE: Object Linking and Embedding

Siemens TIA Portal: Siemens Totally Integrated Automation Portal

\section{ACKNOWLEDGMENT}

We would like to give great thanks to Dr. Greg Arbuckle, our Faculty Advisor for not just overseeing the project but being involved with our project. During the process Dr. Arbuckle was a part of the team, not just someone to answer to when we needed help or found a solution. This project has been a joy to work on and Dr. Arbuckle is one of the main reasons why. We would also like to thank Dr. Doug Harper with his expert advice on LabVIEW. Finally one more acknowledgment should go to Dr. Stacy Wilson who after some administrative changes has taken over as our Faculty advisor when Dr. Arbuckle became Dean of Ogden College. 